Cuadernos de Economía, Vol. 42 (Noviembre), pP. 283-305, 2005

\title{
DiseÑo DE INSTRUMENTOS ECONÓMICOS PARA LA INTERNALIZACIÓN DE EXTERNALIDADES DE ACCIDENTES DE TRÁNSITO
}

\author{
Luis IGNACIO Rizzi* \\ Pontificia Universidad Católica de Chile
}

Road crashes are a major source of transport externalities. Based on Jansson's road crashes model, this paper analyzes ways to internalize those externalities. Jansson's model is extended to analyze the effect of congestion and safety variables related to vehicle and infrastructure design on crash rates and severity. The model is then used to compute the cost of road crashes externalities in Chile.

JEL: $\mathrm{H} 23, \mathrm{H} 29, \mathrm{~K} 13$

Keywords: Externalities, Pigouvian Taxes, Value of Risk Reductions, Road Safety

\section{INTRODUCCIÓN}

Los accidentes de tránsito constituyen una de las externalidades de mayor impacto del sector transporte. Una decisión de un agente económico que afecta el nivel de utilidad o el nivel de producción de otro agente origina una externalidad. Esta se convierte en una falla de mercado si no está adecuadamente internalizada; es decir, si no existen mecanismos que trasladen al generador de la externalidad los costos o beneficios impuestos sobre terceros agentes. Las prácticas actuales en materia de internalización de externalidades de accidentes tanto a nivel internacio-

\footnotetext{
*Un especial agradecimiento para Milton Bertín-Jones, quien motivó parte de este trabajo, leyó una versión preliminar de este documento e hizo valiosas sugerencias. También agradezco los detallados comentarios de un árbitro que ayudaron a mejorar el documento de manera significativa. Por supuesto, todos los errores que permanecen son responsabilidad mía. Por último, agradezco al proyecto MECESUP/PUC 9903 y al Proyecto FONDECYT 1020981 por el financiamiento brindado

*E-mail: lir@ing.puc.cl
} 
nal (Lindberg et al., 1999; Lindberg, 2001) como nacional son imperfectas. La correcta internalización de los efectos externos de los accidentes de tránsito podría tener un importante impacto en materia de prevención de accidentes y, por lo tanto, el diseño y la implementación de instrumentos de internalización deberían ser considerados dentro de una política integral de seguridad de tránsito.

Un vehículo-kilómetro adicional en una vía de circulación causa múltiples externalidades de accidentes. Primero, una unidad extra de tráfico en corrientes de tráfico homogéneas puede modificar la tasa o riesgo de accidentes, afectando la seguridad de todos los conductores. Segundo, existen diferentes tipos de tráfico compartiendo la vialidad, lo que da lugar a accidentes entre miembros de diferentes categorías de tráfico. En este contexto, una unidad adicional de tráfico de cualquiera de las dos categorías puede alterar la tasa de accidentes entre diferentes corrientes de tráfico. Estas dos primeras externalidades son internas al sistema de transporte y dan origen a los costos de accidentes internos al sistema de transporte. Tercero, los accidentes viales generan externalidades externas al sistema de transporte, principalmente a través de daños a la propiedad de terceros, costos de atención médica, etc., y dan origen a los costos de accidentes externos del sistema de transporte y recaen sobre el resto de la sociedad. Cuarto y último, ciertos agentes económicos (empresas automotrices y proveedores de infraestructura) son proveedores de insumos vitales en la producción de unidades de vehículos-kilómetros. En este sentido, se quiere estudiar si el diseño de sus productos conduce o no al nivel óptimo de seguridad vial.

En la literatura, es estándar considerar las externalidades internas y externas al sistema de transporte en un análisis económico sobre prevención de accidentes: Jansson (1994) hizo una contribución seminal y fue seguido por Elvik (1994), Persson y Ödegaard (1995), Fridstrøm (1999), Lindberg et al. (1999) y Lindberg (2001). A partir de estos modelos, se pueden determinar tarifas óptimas de seguros de accidentes viales a fin de internalizar las externalidades del tráfico homogéneo, el tráfico heterogéneo y los impactos sobre el resto de la sociedad.

La primera contribución de esta investigación consiste en estudiar cómo valorar los efectos que la congestión y las políticas de diseño vehicular e infraestructura vial producen en la generación de externalidades de accidentes viales. La congestión puede tener un impacto tanto sobre la frecuencia como la severidad de los accidentes. El primer fenómeno ha sido estudiado en las referencias citadas, no así el impacto en términos de la severidad de los accidentes. Tampoco existe un análisis en la literatura sobre el impacto de las variables de diseño vehicular y diseño vial sobre los costos externos de accidentes. En relación a este último, se analiza si los agentes de la industria disponen de adecuados incentivos para invertir en la seguridad vial de los productos ofrecidos (vehículos y vías). La segunda contribución de este documento consiste en entregar una estimación de los costos externos de los accidentes viales en Chile.

Luego de la Introducción, la segunda sección desarrolla un modelo de costos de accidentes para corrientes de tráfico homogéneo y, en la tercera sección, el modelo se extiende a condiciones de tráfico mixto. En la cuarta sección se propo- 
ne un impuesto pigouviano para la gestión de la seguridad vial y se explica su funcionamiento en detalle. La quinta sección presenta la estimación de los costos externos de accidentes viales en Chile y la sexta sección cierra el artículo con las conclusiones.

\section{Modelo con Corrientes Homogéneas de Tráfico Vehicular}

Se supone una red vial en la que cada vehículo tiene un riesgo $r$ de sufrir un accidente por unidad de tiempo. El riesgo $r$ es una función del total de vehículoskilómetros circulados por unidad de tiempo, $Q,{ }^{1} \mathrm{y}$ de dos variables cualitativas, $q_{\alpha}$, que contribuyen a evitar accidentes y están relacionadas con el diseño vial (i), $q_{\alpha i}$, y el diseño del vehículo $(v), q_{\alpha v}$. Así, $r=r\left(Q, q_{\alpha i}, q_{\alpha v}\right)$ tiene derivada primera no positiva y derivada segunda no negativa con respecto a $q_{\alpha}$. Los términos vehículo-kilómetro o tráfico se usan indistintamente. También se supone una relación uno a uno entre vehículos y conductores.

Dado un accidente, existe una probabilidad condicionada de que el mismo derive en una víctima fatal o en una víctima herida de gravedad. Esta probabilidad es función de dos variables cualitativas, $q_{f}$, una vez más vinculadas al diseño vial y diseño del automóvil, $q_{f i}$ y $q_{f v}$ respectivamente. Se supone que la proporción de accidentes fatales, $F$, es una función (débilmente) decreciente en estas dos variables, con derivadas segundas no negativas; también es función del volumen de tráfico $Q$. El resto de los accidentes genera víctimas graves, $H(=1-F)$. La modelación explícita de la ocurrencia de víctimas fatales o víctimas graves en un accidente de tránsito es un aporte del presente trabajo, que lo diferencia de aquellos citados en la introducción.

El fenómeno de compensación de riesgos (Peltzman, 1975), tema que suscita mucha atención en materia de seguridad vial, ${ }^{2}$ sugiere que los conductores ajustan su comportamiento ante un cambio en las variables $q_{\alpha j,} q_{f j}(j=i, v)$, de manera tal que el efecto final de una mejora de seguridad es inferior al originalmente previsto. Fridstrøm (1999) provee una justificación microeconómica. Un conductor maximiza la utilidad de realizar un viaje en automóvil. Este viaje contiene algunos rasgos que le otorgan utilidad, tal como la velocidad de conducción, ya sea por reducir el tiempo de viaje, por el placer de la velocidad o simplemente por combatir el aburrimiento. Así, la persona elige una combinación riesgo-velocidad óptima. Ante una mejora en la seguridad, existirá un incentivo a conducir más rápido, de modo que el efecto final sobre la seguridad será menor que el previsto ceteris paribus.

\footnotetext{
${ }^{1}$ El valor de $Q$ se determina a partir de la decisión de los conductores de cuánto viajar en automóvil, que depende de los costos generalizados de viaje. A los efectos del modelo, $Q$ es un dato. Ver Figura 1 y el párrafo que la precede en la sección 4.

${ }^{2}$ Se recomiendan las lecturas de OECD (1990), Fridstrøm (1999) y Wilde (2000)
} 
Qué tan fuerte es el fenómeno de la compensación de riesgo es una cuestión empírica. Para los efectos del presente análisis, supondremos que no existe un comportamiento compensatorio de tal magnitud que empeore la situación en materia de seguridad y, así, se justifican los signos de las derivadas de las funciones $r$ y $F$. En particular, en países en vías de desarrollo, creemos que hay un gran margen aún para medidas ingenieriles de seguridad, especialmente en cuanto a diseño de infraestructura vial. De todas maneras, el modelo puede ser adaptado a fin de incluir comportamientos compensatorios haciendo positivas las derivadas de $F$ y $r$ respecto a las variables $q$.

El costo total de un accidente incluye los costos internos y los costos externos al sistema de transporte. Por costos internos ${ }^{3}$ al sistema de transporte nos referimos a aquellos costos incurridos por los usuarios de este sistema. En primer lugar, incluimos la disposición a aceptar compensación (a pagar) en el margen ante un aumento (disminución) en el nivel de riesgo por efecto de las externalidades de accidentes producidas por una unidad adicional de tráfico. Supongamos un individuo que posee la siguiente función de utilidad esperada, $U E=$ $(1-r)^{*} U(I)$, donde $U$ es utilidad e $I$ es ingreso. Si $r=r(Q, \bullet, \bullet)$, con derivada respecto a $Q$ distinta de cero (positiva o negativa), existirá una externalidad (negativa o positiva) puesto que la utilidad esperada del individuo dependerá de un factor que no está bajo su control, el tráfico vehicular. Dado que la seguridad vial es un bien público, estas disposiciones al pago deben ser agregadas sobre todos los individuos afectados y este valor puede ser aproximado por el promedio de las tasas marginales de sustitución entre ingreso y riesgo sobre la población afectada, llamado en la literatura Valor de las Reducciones de Riesgo (VRR) (Jones Lee y Loomes, 2003) o de manera algo desafortunada el valor de la vida estadística (Rizzi y Ortúzar, 2003). ${ }^{4}$

En segundo lugar, se tienen los costos materiales o médicos asumidos por las víctimas. La suma de estos costos y el VRR arrojan el total de los costos internos y los denominaremos $a_{m}$ y $a_{g}$ según se trate de un accidente con una víctima fatal o una víctima grave respectivamente. El VRR es el principal componente de estos valores; en caso de accidentes fatales, puede constituir más del $90 \%$ del total del valor de $a_{m}$ (Lindberg et al., 1999, Cuadro A.3).

Los costos externos comprenden daños a propiedad de terceros, atención médica y hospitalaria, costos de policía, gastos administrativos y judiciales, y el valor actual neto del costo para las arcas del estado por pérdida de contribuciones impositivas de los damnificados; denominaremos a estos $\operatorname{costos} c_{m}$ y $c_{g}$ para los casos de accidentes fatales y accidentes con víctimas graves, respectivamente. El monto de los costos externos depende de los arreglos institucionales de cada país. Por ejemplo, si la víctima de un accidente de tráfico debe hacerse cargo de los

\footnotetext{
${ }^{3}$ De aquí en más, costo interno o costo externo equivaldrá a decir costo interno del sistema de transporte o costo externo del sistema de transporte.

${ }^{4}$ El VRR depende de manera explícita de la actitud frente al riesgo de los conductores. Cuanto más aversos al riesgo sean, mayor será el VRR.
} 
costos de atención hospitalaria, estos costos serán internos al sistema de transporte, en lugar de externos.

El modelo planteado en esta investigación, que es una extensión de Jansson (1994), considera explícitamente la ocurrencia de accidentes fatales y no fatales en función, entre otras variables, de $Q$. El costo total se expresa así:

$$
C T=\left\{\left(a_{m}+c_{m}\right) F+\left(a_{g}+c_{g}\right) H\right\} r Q
$$

El número total de accidentes, $A$, es igual a $r Q / n$, donde $n$ representa el número promedio de automóviles por accidente. Nótese que $C T=\left\{\left(a_{m}+c_{m}\right) F+\left(a_{g}+c_{g}\right) H\right\} r Q=\left\{\left(a_{m}+c_{m}\right) F+\left(a_{g}+c_{g}\right) H\right\} n A$. La última igualdad pone de manifiesto que los costos esperados o ex ante de accidentes son iguales a los costos reales o ex post de accidentes. Nótese que el vector $[F r Q$; $H r q]$ representa la función de producción de accidentes fatales y graves, respectivamente.

Si se escribe la ecuación de costo total de esta manera alternativa:

$$
C T=\left\{\left(a_{m}+c_{m}\right)-\left(a_{g}+c_{g}\right)\right\} F r Q+\left(a_{g}+c_{g}\right) r Q
$$

todos los accidentes tienen un costo base, $a_{g}+c_{g}$, y a los accidentes fatales hay que adicionarles un costo extra. La importancia de reducir la proporción de accidentes fatales consiste en evitar este costo adicional, suponiendo $\left\{\left(a_{m}+c_{m}\right)-\left(a_{g}+c_{g}\right)\right\}$ mayor a cero. De esta manera, debería ser transparente para el lector la ventaja de modelar de manera explícita el tipo de víctima que un accidente genera.

El costo marginal de accidentes asociado a un kilómetro-vehículo extra es

$$
\begin{aligned}
\frac{\partial C T}{\partial Q} & =\left\{\left(a_{m}+c_{m}\right) F+\left(a_{g}+c_{g}\right)(1-F)\right\} r\left(E_{r}^{Q}+1\right) \\
& +\left\{\left(a_{m}+c_{m}\right)-\left(a_{g}+c_{g}\right)\right\} r F E_{f}^{Q}
\end{aligned}
$$

donde $E_{r}^{Q}$ es la elasticidad del riesgo de accidente en relación al tráfico y $E_{f}^{Q}$ es la elasticidad del riesgo fatal en relación al tráfico. La ecuación anterior se diferencia de expresiones equivalentes encontradas en la literatura, justamente, por la aparición del segundo sumando del lado derecho, que refleja el efecto de la congestión sobre la gravedad de los accidentes, información contenida en $E_{f}^{Q}$.

Desde el punto de vista individual, cada conductor percibe un costo igual al costo medio $\left(C M=\left\{a_{m} F+a_{g} H\right\} r Q / Q=\left\{a_{m} F+a_{g} H\right\} r\right)$; es decir, el conductor percibe los costos que recaen sobre él, no así los costos que impone al resto de la sociedad. Por lo tanto, si al costo marginal se le resta el costo medio percibido por el conductor por kilómetro transitado se obtiene la cuantía de los efectos externos no percibidos, diferencia que constituye un cargo a cobrar a los conductores. 
Podemos expresar este diferencial, desglosando según se trate de un costo interno o externo al sistema de transporte, así:

$$
\begin{aligned}
\frac{\partial C T}{\partial Q}-C M & =\left\{a_{m} F+a_{g}(1-F)\right\} r E_{r}^{Q}+\left\{c_{m} F+c_{g}(1-F)\right\} r\left(E_{r}^{Q}+1\right)+ \\
& +\left\{a_{m}-a_{g}\right\} r F E_{f}^{Q}+\left\{c_{m}-c_{g}\right\} r F E_{f}^{Q}
\end{aligned}
$$

El valor de $E_{r}^{Q}$ refleja la relación existente entre un mayor nivel de tráfico y el riesgo de accidentes. En tráfico interurbano, este valor suele ser considerado igual a cero y en tráfico urbano, superior a cero (Lindberg, 2001). En tráfico urbano, SNRA (1989) utiliza valores entre 0,2 y 0,45 . El valor de $E_{f}^{Q}$ es usualmente considerado negativo: a medida que se incrementa el tráfico, se debe conducir a una menor velocidad y, en consecuencia, la gravedad de los accidentes disminuye con la consiguiente reducción de víctimas fatales. Fridstrøm et al. (1995) reportan un valor de $-0,36$ para Suecia y Fridstrøm (1999), valores de $-0,23$ y $-0,06$ para Noruega. Si bien estos valores son estimados con una muestra que no discrimina entre tráfico urbano e interurbano, es muy probable que los datos para altas densidades de tráfico correspondan a áreas urbanas y que sea la influencia de estos datos los que determinan los valores mencionados.

Si ambas elasticidades son igual a cero, caso probable en tráfico vial interurbano, la expresión (4) se reduce a $\left\{c_{m} F+c_{g}(1-F)\right\} r$ y corresponde cobrar una tarifa que cubra los costos esperados externos de los accidentes. Para el caso de tráfico urbano, los dos primeros sumandos en la ecuación (4) son positivos y los últimos dos dependen de la relación entre tráfico y riesgo de accidentes, esperándose que toda la ecuación sea positiva, excepto en casos de muy severa congestión, como explicamos a continuación.

Si el nivel de tráfico crece de tal forma que la densidad vehicular se vuelve muy alta, los costos de los accidentes en términos de vidas humanas o de víctimas graves serán cada vez menores, debido a las cada vez menores velocidades de circulación. Ocurrirán, entonces, accidentes cuyos costos representarán en su mayoría daños a los vehículos como consecuencia de accidentes leves. Analíticamente, es el segundo sumando del lado derecho de la ecuación (3) el que permite apreciar la disminución de costos por transformar accidentes fatales en accidentes graves. En rigor, se debería haber considerado una tercera categoría de víctimas, que incluyera víctimas leves o víctimas sin consecuencias. La alta congestión supone que la mayoría de las víctimas de accidentes viales pertenecen a esta tercera categoría. En Suecia, el costo de una víctima grave representa el 16 por ciento del costo del VRR y una víctima leve, menos del 0,7 por ciento del VRR (Lindberg et al., 1999; Cuadro A.3); Jones Lee et al. (1993) calculan el costo de víctimas graves en un ocho por ciento del costo de una víctima fatal. Así, reducir la gravedad de los daños físicos a las personas produce un ahorro de costos significativo. Según Fridstrøm (1999), la congestión podría ser un factor que contribuye a las bajas tasas de accidentalidad actuales en áreas congestionadas de los países de Europa noroccidental. 
Estudiemos los efectos de las cuatro variables cualitativas $q_{\alpha i}, q_{\alpha v}, q_{f i}, q_{f v}$ sobre los costos de accidentes. En primer lugar, consideremos las variables que previenen accidentes $q_{\alpha i}, q_{\alpha \nu}$ :

$$
\frac{\partial C T}{\partial q_{\alpha j}}=\frac{\partial r}{\partial q_{\alpha j}} Q\left\{\left(a_{m}+c_{m}\right) F+\left(a_{g}+c_{g}\right)(1-F)\right\} \quad j=i, v
$$

La ecuación (5) indica que existe un beneficio marginal asociado a una mejora en la calidad de los atributos $q_{\alpha i}, q_{\alpha \nu}$ y está dado por el producto de tres factores: a) el costo de una víctima fatal y una víctima grave, cada uno ponderado por su proporción en el total de víctimas, b) el total de vehículos-kilómetros por unidad de tiempo y c) el efecto marginal que el atributo cualitativo tiene sobre la prevención de accidentes. Si el valor de c) fuese igual a cero, no habría nada que la ingeniería vial y/o la ingeniería de diseño vehicular puedan hacer para disminuir el costo de accidentes.

En segundo lugar, estudiemos el impacto de las variables que reducen la gravedad de los daños físicos a las personas en caso de accidente, $q_{f i}, q_{f v}$ :

$$
\frac{\partial C T}{\partial q_{f j}}=\frac{\partial F}{\partial q_{f j}} r Q\left\{\left(a_{m}+c_{m}\right)-\left(a_{g}+c_{g}\right)\right\} \quad j=i, v
$$

Existe un beneficio marginal asociado a mitigar los impactos de un accidente tal que se reduzcan las fatalidades y está dado por el producto de tres elementos: a) el diferencial de costos entre una víctima fatal y una víctima grave, b) el número total de vehículos accidentados $(r Q=A n)$ y c) el efecto marginal del atributo cualitativo sobre la disminución de víctimas fatales. Una vez más, si este último elemento fuese igual a cero, no habría posibilidades de reducir los costos de los accidentes fatales.

El nivel óptimo de provisión de $q_{\alpha i}, q_{\alpha v}, q_{f i}, q_{f v}$ corresponde al valor en que se igualan el beneficio marginal y el costo marginal de una mejora en cada una de dichas variables. La interrogante es la siguiente: ¿Están dadas las condiciones para la provisión óptima de las variables $q_{\alpha i}, q_{\alpha v}, q_{f i}, q_{f v}$ ? En principio sí, siempre y cuando los conductores posean conocimiento perfecto sobre la eficacia de los implementos de seguridad vial. En el caso de la compra de un vehículo, los conductores elegirían los automóviles que brindasen el nivel de seguridad óptimo individual y ésta sería una señal suficiente para que las empresas automotrices produzcan vehículos con los niveles de seguridad deseados por sus clientes.

Si los conductores no pudiesen reconocer la eficacia de los implementos de seguridad, ninguna firma dispondría de incentivos adecuados para invertir recursos extra en medidas de seguridad, puesto que no podrían incorporar estos costos en el precio final del vehículo. En este caso, el nivel de seguridad ofrecido por los vehículos sería inferior al óptimo. Peor aún, el valor de la ecuación (1) no estaría calculado en el valor óptimo del vector $q\left(q^{\text {opt }}\right)$, sino que en un valor diferente (por ejemplo, $q^{0}$ ) incrementando la cuantía de los costos totales. Sin embargo, el 
establecimiento del impuesto pigouviano solucionaría este problema, puesto que su valor dependerá del vector de atributos $q$ que corresponda al automóvil utilizado. De esta forma, existiría una señal de precios que los automovilistas percibirían, la que finalmente induciría a los agentes de la industria a mejorar la seguridad de los vehículos.

También podría suceder que los individuos perciban de manera imperfecta los riesgos de accidente. En principio, cabría esperar que la incorrecta percepción de riesgos no presente un sesgo definido; en otras palabras, habría conductores que sobreestimarían el riesgo de accidente y otros que lo subestimarían. En el primer caso habría una propensión a demandar mayor seguridad vehicular que la socialmente óptima y, en el segundo, se demandaría menor seguridad vial que la óptima. Una vez más, el nivel de provisión de mercado de seguridad vial diferirá del óptimo social.

En el caso de la infraestructura vial, la respuesta sobre la factibilidad de alcanzar el nivel óptimo de seguridad vial es aún más compleja: no sólo cuentan las razones recién esgrimidas, sino que deben considerarse otras dos complicaciones. En primer lugar, la provisión y operación de la infraestructura vial suele poseer la característica de un monopolio natural. En segundo lugar, la seguridad vial adquiere la dimensión de no rivalidad: una mejora de diseño vial con impacto positivo en la seguridad vial estará disponible para todos los usuarios de la vialidad. Nuevamente, es difícil suponer que el mercado proveerá condiciones óptimas de seguridad en relación a la infraestructura vial.

\section{Modelo con Corrientes Mixtas de Tráfico}

En esta sección, una vez más seguimos a Jansson (1994) en el tratamiento de un caso más complejo: la existencia de tráficos mixtos. A fin de simplificar la notación, se utiliza como ejemplo el caso de vehículos y bicicletas, aunque es fácil la extensión a otras categorías mixtas de tráfico. ${ }^{5}$ Se supone que $X$ es el número de accidentes entre vehículos y bicicletas, $M$ el número total de bicicleta - kilómetros. ${ }^{6}$ Se supone que en cada accidente ${ }^{7}$ participan un ciclista y un automovilista y que por cada accidente entre automóvil y bicicleta habrá sólo una víctima. La víctima (fatal o grave) será un ciclista con una probabilidad $\theta$ y será un automovilista (fatal o grave) con una probabilidad $1-\theta$.

Una vez más, modelamos de manera explícita la probabilidad de ocurrencia de una víctima fatal o una víctima grave como consecuencia de un accidente de tránsito. Tanto la ocurrencia de accidentes como la distribución de las víctimas

\footnotetext{
${ }^{5} \mathrm{Si}$ se consideran las categorías de tráfico $z$ y $w$, la palabra automóvil se reemplaza por la categoría de tráfico $z$ y la palabra bicicleta, por la categoría de tráfico $w ; z$ y $w$ representan dos cualesquiera categorías de tráfico.

${ }^{6} \mathrm{El}$ valor de $M$ se determina de manera similar a $Q$; es decir, $M$ y $Q$ son valores dados a los efectos del modelo simplificado de accidentes. Ver nota al pie 1.

${ }^{7}$ En esta sección, la palabra accidentes se refiere a accidentes entre automovilistas y ciclistas.
} 
entre fatales y no fatales dependen de las variables $q_{\alpha i}, q_{\alpha v}, q_{f i}, q_{f v}$. Se adicionan las variables de calidad $q_{\alpha b}, q_{f b}$, relacionadas con la seguridad de las bicicletas en términos de prevención de accidentes y de mitigación de los efectos de accidentes. La función de riesgo de accidente para usuarios de bicicletas es $r_{b}=r_{b}\left(Q, M, q_{\alpha i}, q_{\alpha v}, q_{a b}\right)$, la función de riesgo de accidente para usuarios de vehículos es $r_{v}=r_{v}\left(Q, M, q_{\alpha i}, q_{\alpha v}, q_{a b}\right), r_{b}=X / M, r_{v}=X / Q$ y las funciones de riesgo condicionado de accidentes fatales para usuarios de bicicletas y vehículos respectivamente son $F_{b}=F_{b}\left(Q, M, q_{f i}, q_{f v}, q_{f b}\right)$ y $F_{v}=F_{v}\left(Q, M, q_{f i}, q_{f v}, q_{f b}\right)$. En este caso, las variables $q_{f v} \mathrm{y} q_{f b}$ incluyen también elementos de protección hacia la otra categoría de tráfico: por ejemplo, parachoques amigables que, en caso de impacto, produzcan un menor daño sobre un ciclista (o peatón) que un parachoques convencional. Aún más, $\theta$ podría considerarse función de (un subconjunto) las variables de diseño.

El costo total de los accidentes está dado por la siguiente expresión:

$$
\begin{aligned}
C T= & \left\{\left(a_{m}+c_{m}\right) F_{b}+\left(a_{g}+c_{g}\right) H_{b}\right\} r_{b} M \theta+ \\
& +\left\{\left(a_{m}+c_{m}\right) F_{v}+\left(a_{g}+c_{g}\right) H_{v}\right\} r_{v} Q(1-\theta) \\
C T= & C T^{M} \theta+C T^{Q}(1-\theta)
\end{aligned}
$$

Derivando con respecto a $Q$ y $M$, se obtienen los costos marginales de agregar a la red un automóvil-kilómetro y una bicicleta-kilómetro extra.

$$
\begin{aligned}
\frac{\partial C T}{\partial Q} & =\frac{C T^{M}}{Q} E_{r_{b}}^{Q} \theta+\left\{\left(a_{m}+c_{m}\right) F_{v}+\left(a_{g}+c_{g}\right)\left(H_{v}\right)\right\}_{v}\left(1+E_{r_{v}}^{Q}\right)(1-\theta)+ \\
& +\left\{\left(a_{m}+c_{m}\right)-\left(a_{g}+c_{g}\right)\right\}\left(E_{F_{b}}^{Q} r_{b} F_{b} \frac{M}{Q} \theta+E_{F_{v}}^{Q} r_{v} F_{v}(1-\theta)\right) \\
\frac{\partial C T}{\partial M} & =\left\{\left(a_{m}+c_{m}\right) F_{b}+\left(a_{g}+c_{g}\right)\left(H_{b}\right)\right\} r_{b}\left(1+E_{r_{b}}^{M}\right) \theta+\frac{C T Q}{M} E_{r_{v}}^{M}(1-\theta)+ \\
& +\left\{\left(a_{m}+c_{m}\right)-\left(a_{g}+c_{g}\right)\right\}\left(E_{F_{b}}^{M} r_{b} F_{b} \theta+E_{F_{v}}^{M} r_{v} F_{v} \frac{Q}{M}(1-\theta)\right)
\end{aligned}
$$

El costo marginal de adicionar un vehículo-kilómetro a la red está relacionado con tres elementos: a) la elasticidad del riesgo de accidente en relación al tráfico vehicular para el usuario de bicicleta, b) la elasticidad del riesgo de accidente para el usuario de automóvil en relación al tráfico vehicular y c) las elasticidades del riesgo de accidente fatal para el usuario de bicicleta y el usuario de vehículo en relación al tráfico vehicular. ${ }^{8}$ El primer elemento dice relación sobre el efecto de

${ }^{8}$ Este término refleja explícitamente el efecto de la congestión sobre la gravedad del accidente y diferencia a la ecuación (8) de expresiones equivalentes obtenidas en otras referencias. 
adicionar un vehículo-kilómetro a la red sobre el riesgo de accidente entre un automóvil y una bicicleta para los usuarios de bicicleta; el segundo elemento, sobre el efecto de agregar un vehículo-kilómetro sobre el riesgo de accidente entre usuarios de automóvil y de bicicletas para los usuarios de vehículos y el último, sobre el efecto que un vehículo-kilómetro adicional tiene sobre la proporción de accidentes fatales. Con las adecuadas modificaciones, la misma interpretación vale para el cálculo del costo marginal de una bicicleta-kilómetro.

De aquí en adelante, se adopta un valor de $\theta$ igual a uno (1), basado en Lindberg (2001). ${ }^{9}$ Esto supuesto implica la existencia de una categoría fuerte de tráfico, el victimario, y una categoría débil, la víctima, que se explica por la diferencia de masas de cada uno de los vehículos involucrados en una colisión. Además, supondremos independencia de la proporción de accidentes fatales y no fatales respecto a los volúmenes de tráfico, tal que $F_{b}=F_{b}\left(q_{f i}, q_{f v}, q_{f b}\right)$ y $F_{v}=F_{v}\left(q_{f i}, q_{f v}, q_{f b}\right)$. De esta manera, la ecuación (8) se transforma en la ecuación (9):

$$
\begin{aligned}
& \frac{\partial C T}{\partial Q}=\frac{C T^{M}}{Q} E_{r_{b}}^{Q} \\
& \frac{\partial C T}{\partial M}=\left\{\left(a_{m}+c_{m}\right) F_{b}+\left(a_{g}+c_{g}\right)\left(H_{b}\right)\right\} r_{b}\left(1+E_{r_{b}}^{M}\right)
\end{aligned}
$$

Para lograr el resultado óptimo, los conductores de automóvil deberían pagar una tarifa equivalente al diferencial entre el costo marginal y el costo medio percibido de su actividad. Este último resulta ser cero, ya que ellos no sufren las consecuencias económicas de los accidentes; entonces la tarifa a cobrarles es igual a $\partial C T / \partial Q$. Por el contrario, los ciclistas perciben un costo medio igual a $\left(a_{m} F_{b}+a_{g} H_{b}\right) r_{b}$ y, por lo tanto, deberían pagar una tarifa equivalente a

$$
\begin{aligned}
\frac{\partial C T}{\partial M}-C M & =\left\{\left(a_{m}+c_{m}\right) F_{b}+\left(a_{g}+c_{g}\right) H_{b}\right\} r_{b} E_{r_{b}}^{M}+\left\{c_{m} F_{b}+c_{g} H_{b}\right\} r_{b} \\
& =C T^{M} \frac{E_{r_{b}}^{M}}{M}+\left\{c_{m} F_{b}+c_{g} H_{b}\right\} r_{b}
\end{aligned}
$$

Finalmente, la recaudación total sería igual a la suma de los dos componentes mencionados, en las ecuaciones (9) y (10), multiplicados por $Q$ y $M$ respectivamente:

$$
R T=C T^{M} E_{r_{b}}^{Q}+C T^{M} E_{r_{b}}^{M}+\left\{c_{m} F_{b}+c_{g} H_{b}\right\}_{b} M
$$

\footnotetext{
${ }^{9}$ Lindberg (2001, Cuadro 4) muestra posibles valores de $\theta$ para distintas categorías de tráfico mixto. En el caso de colisiones entre automovilistas y ciclistas (usuarios desprotegidos en el Cuadro 4), dicho valor es 0,99 .
} 
El primer sumando del lado derecho de la ecuación representa el monto (la compensación) total que deberían pagar (recibir) los automovilistas a fin de internalizar sus externalidades de accidentes en corrientes de tráfico mixto. El segundo sumando representa el mismo fenómeno para los ciclistas. En la medida que $E_{r_{b}}^{Q} y E_{r_{b}}^{M}$ sean simultáneamente distintos de cero, cada corriente de tráfico estará generando un externalidad interna al sistema de transporte (positiva o negativa). El tercer sumando comprende los costos externos que los accidentes entre corrientes heterogéneas de tráfico imponen sobre el resto de la sociedad.

$\operatorname{Si}\left(E_{r_{b}}^{Q}+E_{r_{b}}^{M}\right)$ es igual a cero, la cifra recaudada será igual al costo total esperado externo de los accidentes. Bajo el supuesto que la función de accidentes entre vehículos y bicicletas fuese del tipo Cobb-Douglas en el tráfico de vehículos-kilómetros y bicicleta-kilómetros y homogénea de grado uno, un caso probable sería aquel en que $E_{r_{h}}^{Q}$ y $E_{r_{h}}^{M}$ toman valores de 0,5 y $-0,5$ respectivamente (Jansson, 1994). En este caso, los automovilistas pagan la mitad del costo esperado total de los accidentes; los ciclistas, por un lado, reciben una compensación igual a la mitad del costo total esperado de los accidentes y, por otro lado, han de hacerse cargo de los costos externos de los accidentes en los que se ven involucrados. La presencia de un ciclista es necesaria para la ocurrencia de un accidente ${ }^{10} \mathrm{y}$ al convertirse en una víctima de accidente, impone un costo externo, que debe ser internalizado. El subsidio neto recibido por los ciclistas es igual a la diferencia entre el pago total de los conductores y el valor esperado de los costos externos. Dado que los costos internos de accidentes fatales y graves valorados según la disposición a aceptar compensación son muy superiores a los costos externos de los accidentes fatales y graves, los ciclistas serán efectivamente subsidiados. En caso contrario, ellos tendrán que afrontar un pago positivo.

Concluir que, bajos ciertos supuestos, los ciclistas deben ser subsidiados estaría en contradicción con el resultado típico en modelación de externalidades que sostiene que las víctimas no deben ser compensadas (Baumol y Oates, 1988). Sin embargo, los ciclistas no son subsidiados por ser víctimas, sino que por aportar el insumo bicicleta-kilómetro, que contribuye a disminuir el riego de accidentes en la función de producción de accidentes. En otras palabras, el subsidio tiene por finalidad alentar el uso de la bicicleta y no compensar el daño sufrido. Así, reconciliamos nuestra postura con el resultado clásico de la teoría económica sobre externalidades.

Pasemos a investigar los costos externos vinculados con las variables $q_{a j}$ y $q_{f f}$; derivando en relación a ellas se obtienen las siguientes expresiones:

$$
\frac{\partial C T}{\partial q_{\alpha j}}=\frac{\partial r_{b}}{\partial q_{\alpha j}} M\left\{\left(a_{m}+c_{m}\right) F_{b}+\left(a_{g}+c_{g}\right)\left(1-F_{b}\right)\right\} \quad j=i, v, b
$$

\footnotetext{
${ }^{10} \mathrm{El}$ ciclista es parte de la función de producción de accidentes entre vehículos y ciclistas.
} 


$$
\frac{\partial C T}{\partial q_{f j}}=\frac{\partial F_{b}}{\partial q_{f j}} r_{b} M\left\{\left(a_{m}+c_{m}\right)-\left(a_{g}+c_{g}\right)\right\} \quad j=i, v, b
$$

Las ecuaciones (12) y (13) entregan el efecto de una mejora en las variable genéricas $q_{a j}$ y $q_{f j}$ sobre el costo total de los accidentes, respectivamente, y se interpretan de manera similar a las ecuaciones (5) y (6). El caso de tráfico mixto incorpora la existencia de elementos de diseño vehicular con efecto positivo sobre la seguridad del modo bicicleta.

El análisis correspondiente a las ecuaciones (5) y (6) sobre la factibilidad que el mercado provea los niveles óptimos de seguridad vial se aplica nuevamente en este caso, pero debemos añadir una consideración adicional que vuelve este caso más complejo. Pensemos en aquellas variables de seguridad vial a implementar en los vehículos (categoría fuerte de tráfico) cuyo efecto en términos de seguridad sólo beneficia a los ciclistas (categoría débil de tráfico). En este caso, estamos en la presencia de una externalidad positiva, puesto que no beneficia al usuario del automóvil, quien ha de pagar por el implemento de seguridad beneficioso para terceros. Según indica la teoría, el consumo de un bien que genera una externalidad positiva es inferior al óptimo social; peor aún, en este caso dicho bien no sería demandado en absoluto. Así, las ecuaciones en (9) serían calculadas en un valor $q^{0}$ diferente al valor óptimo $q^{\text {opt }}$. En este caso, el establecimiento de una tarifa igual a $\frac{\partial C T}{\partial Q_{k q^{0}}}$ provee la señal necesaria para que el victimario incorpore los costos externos que éste genera sobre la categoría débil de tráfico. El conductor del automóvil hallará conveniente disponer de un automóvil más amigable con los usuarios de bicicletas y pagar un menor impuesto pigouviano. ${ }^{11}$ De lo contrario, pagará en concepto de impuesto pigouviano una cantidad igual a todo el beneficio social perdido como producto de su decisión no óptima, que claramente ha de ser un valor superior al costo total de pasar de $q^{0}$ a $q^{\text {opt }}$.

\section{Impuestos Pigouvianos para la Gestión de la Seguridad Vial}

En esta sección, analizaremos el uso de un instrumento de mercado que permita lograr una mejor gestión de seguridad vial. En particular, nos centraremos en el uso de precios, dejando de lado instrumentos de regulación como ser estándares de seguridad, elementos indispensables en una política de seguridad de tránsito que procure acercarse al óptimo social (Shavell, 1984, 2003).

El instrumento de gestión propicio para lograr una adecuada internalización de los costos de externalidades de accidentes es el cobro de un seguro obligatorio

${ }^{11}$ Un caso peor es aquél en el que una mejora en la seguridad de un vehículo resultase en un menor nivel de seguridad para un tercer vehículo, tal como señala Tay (2002) en el caso de los SUV. Una vez más, la introducción de un impuesto pigouviano que incorpore este efecto externo solucionaría el problema. 
de accidentes (o tarifa vial) por kilómetro circulado. ${ }^{12}$ Para calcular dicha tarifa o impuesto pigouviano, se deben considerar los costos de todos los posibles accidentes entre diferentes categorías de vehículos. Considerando $N$ categorías de tráfico, los costos totales de accidentes del sistema de transporte vial (CTS) estarían dados por la siguiente ecuación:

$$
C T S=\sum_{n=1}^{N} \sum_{j \geq n}^{N} C T(n, j)
$$

En la ecuación (14), si las categorías de tráfico coinciden $(n=j)$, se trata de accidentes entre corrientes de tráfico homogéneas; caso contrario $(n \neq j)$, se trata de accidentes entre corrientes mixtas de tráfico. Tal como se procedió anteriormente, los valores clave para establecer la tarifa a cobrar son el costo marginal por kilómetro circulado y el costo medio percibido (CMP) por kilómetro circulado para cada categoría de tráfico $n$ :

$$
\begin{gathered}
\frac{\partial C T S}{\partial Q_{n}}=\frac{\partial \sum_{j=l}^{N} C T(n, j)}{\partial Q_{n}}, \quad \forall n \\
C M P_{n}=\frac{\sum_{j=1}^{N}\left\{a_{m} F_{n}+a_{g} H_{n}\right\} r_{n, j} Q_{n} \theta_{n, j}}{Q_{n}}, \quad \forall n
\end{gathered}
$$

En estas dos ecuaciones, $Q_{n}$ representa el término vehículos-kilómetros de la categoría de tráfico $n$. En la segunda ecuación, la notación fue adaptada de la siguiente manera: $r_{n j}$ es el riesgo para la clase $n$ de sufrir un accidente con un vehículo de la clase $j$ y $\theta_{n, j}$ es la probabilidad que la víctima de un accidente entre las categorías de tráfico $n$ y $j$ corresponda a la clase $n$, tal que $\theta_{n, j}+\theta_{j, n}=1$. Restando la ecuación (16) a la ecuación (15) se obtiene la tarifa o impuesto pigouviano $\left(t_{n}\right)$ a cobrar por externalidades de accidentes por vehículo-kilómetro circulado por categoría de tráfico:

$$
t_{n}=\frac{\partial C T S}{\partial Q_{n}}-C M P_{n}, \quad \forall n
$$

Esta tarifa discrimina por categoría de tráfico y por la intensidad de uso vehicular. En rigor, la tarifa influirá en la decisión de cuántos vehículo - kilómetros conducir por cada tipo de vehículo o categoría de tráfico. ${ }^{13}$

\footnotetext{
${ }^{12}$ Técnicamente, tarificar según los criterios anteriores es factible mediante el uso de técnicas de verificación de kilometraje circulado por automóvil (Litman, 2004).

${ }^{13} \mathrm{La}$ tarifa tendría que considerar un elemento adicional, no contemplado en el modelo: el historial de conducción en términos de accidentalidad tal que a mejor historial menor sea el cargo a cobrar.
} 
Esta tarifa funcionaría como un cargo ex ante para los conductores tal que, una vez ocurrido el accidente, todo automovilista estaría cubierto en relación a los costos de externalidades que impone sobre el resto de la sociedad. El cobro de este cargo supone el principio de responsabilidad objetiva pura. Quien conduce un vehículo de mayor tamaño es el agresor, independientemente de la existencia o no de negligencia, y el usuario no motorizado o vehículo de menor tamaño es la víctima, independientemente de su culpabilidad. En colisiones entre vehículos de igual categoría, cada uno de los conductores carga con sus respectivos costos y los costos externos se reparten de manera proporcional entre los vehículos que participaron en el accidente, independientemente de quién haya sido culpable del mismo.

Las víctimas de los accidentes que pertenecen a la categoría débil, en corrientes de tráfico mixtas, tendrían que pagar los costos externos al sistema de transporte (sección 2). Desde el punto de vista de las víctimas de las categorías débiles, el sistema funcionaría así: cada una de las potenciales víctimas de las categorías débiles ${ }^{14}$ recibe un subsidio, neto de los costos externos de accidentes, a fin de incentivar su participación en el tráfico (suponiendo que su presencia contribuye a un aumento menos que proporcional en el número de accidentes entre corrientes de tráfico mixtas). Por su parte, los costos externos de accidentes son reembolsados a los terceros agentes que corresponda.

El sistema propuesto tiene por finalidad internalizar los costos de las externalidades de accidentes que recaen sobre terceros agentes económicos, ya sean otros usuarios de vehículos motorizados, usuarios no protegidos o agentes externos al sistema de transporte. Este seguro obligatorio propuesto no otorga cobertura por los costos que recaen sobre el propio automovilista o usuarios desprotegidos. ${ }^{15}$ Para ello, éstos deberían contratar una póliza de seguro adicional que cubra los casos de pérdida personal, ya sea por daños corporales o muerte o daños vehiculares. Eventualmente, podrían permitirse acciones legales posteriores al accidente en casos en que el agresor, además, haya cometido una falta severa (por ejemplo, manejar en estado de ebriedad); ́́dem, en el caso de accidentes entre vehículos de una misma categoría. Para una mayor discusión sobre cuán justo es el sistema propuesto de tarificación ver Shavell (1987), Jansson (1994), Lindberg et al. (1999) y Lindberg (2001).

Es de esperar, en especial, que los usuarios no protegidos no contraten pólizas de seguros en caso de pérdida personal, ya sea por la no disponibilidad de dichos seguros en el mercado, por desconocimiento de cómo operan estos mercados o simplemente por una decisión personal (basada tal vez en una subestimación del riesgo de accidente). Aquí podría pensarse en una variante al sistema propuesto: el monto pagado ex ante por los automovilistas se destina a un fondo cuya finalidad sea cubrir los costos de las víctimas de las categorías débiles de accidentes, en particular peatones y ciclistas. El problema que surge con esta

\footnotetext{
${ }^{14}$ La insistencia en decir víctimas de las categorías débiles se debe a que las víctimas de accidentes entre vehículos de igual categoría no deben ser compensadas.

${ }^{15}$ Los costos que hemos llamado percibidos por el usuario.
} 
variante es que podría desincentivar el tráfico de ciertas categorías de tráfico como los ciclistas, puesto que éstos no recibirían un subsidio ex ante, sino un pago ex post en caso de accidente. Sin embargo, el subsidio desde el punto de vista personal sería una cifra de dinero muy pequeña, que probablemente no afectase de manera significativa el comportamiento de los usuarios de categorías desprotegidas.

Con este tipo de tarificación, se encarecería el uso del automóvil privado para quienes lo utilizan sobremanera o conducen vehículos de mayor masa. Bajo estas condiciones, algunos conductores deberían hacer un uso más limitado del automóvil o pasarse a vehículos de menor masa y algunos pocos, deberían abandonar el uso del automóvil particular. En otras palabras, se haría un uso más racional del automóvil, fin último de este tipo de política.

Finalmente, la introducción de un seguro obligatorio de accidentes también lograría inducir a los fabricantes de automóviles y proveedores de infraestructura vial a dotar a sus productos de niveles óptimos de seguridad. Si las tarifas de los seguros discriminan por el nivel de seguridad que un automóvil brinda a sus pasajeros así como a terceros usuarios, los conductores de automóvil tendrán un incentivo económico para comprar vehículos más seguros, aunque ellos no los perciban como tales. De esta manera, existirá una disposición al pago por una mejor seguridad vehicular que recompensará los recursos invertidos en mayor seguridad por los fabricantes de automóviles.

Más complejo es analizar si esta tarifa puede incentivar diseños viales óptimos. Las empresas automotrices tienen claros incentivos para satisfacer la demanda de sus clientes en la medida que maximicen sus beneficios; por lo tanto, basta con inducir a sus clientes a demandar niveles óptimos de seguridad vial para que ellas actúen en consecuencia. Esta lógica, en principio, se aplicaría también a los proveedores de infraestructura vial, si éstos tuviesen como misión explícita maximizar el bienestar social. Si los proveedores de infraestructura vial no tienen asignado este mandato, no es posible argüir que mediante el cobro de impuestos pigouvianos a los automovilistas se pueda inducir a diseños de infraestructura vial óptimos en términos de seguridad. Este se debe, en parte, al carácter de monopolio natural de la provisión de infraestructura y a que, en la mayoría de los casos, la infraestructura vial es administrada según criterios administrativos. En estos casos, debería contemplarse la posibilidad de un cargo a los proveedores de infraestructura vial que diga relación con cuán lejos están ellos de proveer el nivel óptimo de seguridad vial. Por ejemplo, el monto total del cargo $(P)$ podría ser igual a $P=C T\left(\ldots ; q_{\alpha i}^{0} ; q_{\alpha i}^{0}\right)-C T\left(\ldots ; q_{\alpha i}^{o p t} ; q_{\alpha i}^{o p t}\right)$, donde $q^{0}$ representa el nivel actual de inversión en seguridad vial y $q^{\text {opt }}$, el nivel óptimo. En otras palabras, todo el costo extra de accidentes generado por no proveer el nivel óptimo de seguridad vial debe ser trasladado a la empresa proveedora de la infraestructura vial, que dispondría, entonces, de los incentivos necesarios para proveer el nivel de seguridad óptimo. 


\section{CASO DE APLiCACIÓN}

En esta sección, se calcula el costo actual de las externalidades de accidentes de tránsito en Chile. En relación al monto del VRR a utilizar en los cálculos para valorar víctimas fatales y víctimas graves, adoptaremos un único valor para cada tipo de víctima, independientemente del tipo de vialidad que se trate. ${ }^{16}$ El costo de la víctima fatal o VRR es de \$161 millones, ${ }^{17}$ según Rizzi y Ortúzar (2003); siguiendo a estos autores, el costo de la víctima grave equivale al ocho por ciento del VRR. ${ }^{18}$ Como valor alternativo al VRR, utilizaremos el valor del capital humano, UF 1.250, estimado por CITRA (1996). Excepto que se indique lo contrario, todos los valores están expresados en pesos de mayo de 2004.

Los datos sobre accidentalidad que utilizaremos han sido redondeados para facilitar los cálculos y fueron obtenidos en la Comisión Nacional de Seguridad de Tránsito (CONASET). ${ }^{19}$ Estos incluyen números de accidentes, número de fatalidades, número de heridos graves (agrupándose víctimas graves y muy graves), y proporción de víctimas desprotegidas. El parque vehicular lo supondremos igual a 2.000.000, valor aproximado para el año $2000 .{ }^{20}$ Nos concentraremos sólo en aquellos accidentes que originan víctimas fatales o víctimas graves. Hay muchos otros accidentes, más de 30.000 , que afortunadamente no generan víctimas fatales ni graves; sólo heridos leves o daños materiales; los costos promedios de estos accidentes, tanto en daños materiales como en daños físicos personales, son significativamente inferiores. Del total de 1.700 muertes consideradas y 15.300 víctimas graves, un 40 por ciento corresponde a peatones y ciclistas. El costo interno anual total de estos 17.000 accidentes viales para el país, valorados según el VRR, asciende a la suma de $\$ 471$ mil millones. Si adicionásemos los costos en daños de materiales y los costos externos al sistema de transporte de estos accidentes según CITRA (1996), deberíamos adicionar otros $\$ 94$ mil millones de pesos, sumando un total del $\$ 565$ mil millones.

Para el cálculo de los costos externos de accidentes de tránsito, consideraremos sólo dos categorías de tráfico: conductores de vehículos, que representan la categoría fuerte, y usuarios desprotegidos, peatones en su mayoría, representantes de la categoría débil. Según la evidencia reportada en la sección 2, los conductores deberían pagar la mitad de los costos totales esperados de los atropellos a peatones $\mathrm{y} / \mathrm{o}$ ciclistas. El resto de las muertes corresponden a personas viajando en un vehículo, suponiendo un 50 por ciento en áreas urbanas y otro

\footnotetext{
${ }^{16}$ Este supuesto podría ser incorrecto. Para una discusión al respecto, ver Rizzi y Ortúzar (2005).

${ }^{17}$ Rizzi y Ortúzar (2003) recomiendan un valor conservador de $\$ 142,5$ millones a enero del año 2000. Ajustado por el valor de la UF a fin de mayo de 2004 arroja el valor del texto.

${ }^{18}$ Rizzi y Ortúzar (2003) basan su valor de una víctima grave en Jones Lee et al. (1993).

Trabajo en curso por Hojman et al. (2005) indicaría que el VRR de una víctima grave sería superior al ocho por ciento del VRR de una víctima fatal en Chile.

${ }^{19} \mathrm{http}: / /$ www.conaset.cl/, sección Estadísticas.

${ }^{20}$ El valor exacto para el año 2000 está disponible en www.ine.cl.
} 
50 por ciento en áreas no urbanas. Según nuestros supuestos de la sección 1, estos últimos fallecimientos no generan externalidades internas al sistema de transporte, sino sólo externas. Por su parte, en áreas urbanas se supone que la elasticidad del riesgo de accidente en relación al tráfico es igual a 0,2 e igual a cero en áreas interurbanas; los mismos supuestos cuentan para las víctimas graves en vialidad urbana e interurbana. Suponemos un efecto neutro de la congestión sobre el nivel de riesgo de muerte (condicionado en la ocurrencia de un accidente) en áreas urbanas, dado que Chile aún no tiene los niveles de congestión de tráfico experimentados en muchos países desarrollados. ${ }^{21}$ El Cuadro 1 resume los datos y parámetros relevantes para el cálculo de los costos externos.

CUADRO 1

PARAMETROS Y DATOS RELEVANTES

\begin{tabular}{cc}
\hline & Tráfico \\
Tráfico urbano & interurbano \\
\hline
\end{tabular}

Flujos homogéneos

\begin{tabular}{|c|c|}
\hline$E_{r}^{Q}$ & 0,2 \\
\hline$E_{f}^{Q}$ & 0 \\
\hline
\end{tabular}

Flujos mixtos

$\begin{array}{lrr}E_{r_{b}}^{Q} & 0,5 & 0,5 \\ E_{r_{b}}^{M} & -0,5 & -0,5 \\ \text { Víctimas fatales categorías débiles } & 340 & 340 \\ \text { Víctimas fatales categorías auto } & 510 & 510 \\ \text { Víctimas graves categorías débiles } & 3.060 & 3.060 \\ \text { Víctimas graves categorías auto } & 4.590 & 4.590\end{array}$

El Cuadro 2 entrega la estimación de los costos externos de accidentes viales según el modelo presentado en las secciones 1 y 2 . En la segunda columna, todos los valores de costos (daños personales y daños materiales y consumo de recursos) son tomados de CITRA (1996); en la tercera columna, los costos de daños personales se basan en Rizzi y Ortúzar (2003) y los demás costos, en CITRA (1996). La segunda fila muestra los costos por daños personales por vehículo, suponiendo que un vehículo circula un promedio de $24.611 \mathrm{~km} /$ año. La tercera fila muestra el mismo valor, pero en relación a los costos por daños materiales y consumo de recursos (gastos administrativos, de policia, de salud, etc.). La cuarta fila arroja la suma de ambos costos externos. La quinta, sexta y séptima fila entregan idénticos valores agregando sobre el total del parque vehicular del país.

\footnotetext{
${ }^{21}$ Ver nota al pie 22 para un análisis de sensibilidad al respecto.
} 
CUADRO 2

ESTIMACION DE COSTOS EXTERNOS DE ACCIDENTES VIALES

(\$ de 2004)

\begin{tabular}{lcc}
\hline & Valores CITRA $^{\mathrm{b}}$ & Valores R\&O/CITRA $^{\mathrm{c}}$ \\
\hline Costos daños personales por vehículos $^{\mathrm{a}}$ & $\$ 13.000$ & $\$ 63.500$ \\
Otros costos por vehículos $^{\mathrm{C}}$ & $\$ 33.000$ & $\$ 33.000$ \\
Costos externos totales por vehículo & $\$ 45.800$ & $\$ 96.500$ \\
Costos totales por daños personales $^{\mathrm{d}}$ & $\$ 26$ mil millones & $\$ 127$ mil millones \\
Costos totales por otros costos $^{\text {a, d }}$ & $\$ 66$ mil millones & $\$ 66$ mil millones \\
Costos totales $^{\mathrm{d}}$ & $\$ 92$ mil millones & $\$ 193$ mil millones \\
\hline
\end{tabular}

${ }^{\mathrm{a}}$ Incluye daños materiales y consumo de recursos. ${ }^{\mathrm{b}}$ Los cálculos en esta columna se basan en

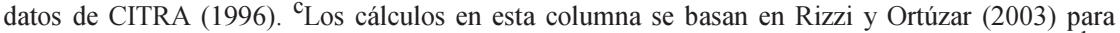
la estimación de daños personales y en CITRA (1996) para la estimación de otros costos. ${ }^{\mathrm{d}} \mathrm{Se}$ supone un parque vehicular igual a dos millones de vehículos.

El monto de los costos totales es muy sensible al valor utilizado para evaluar los costos en daños personales; este monto se incrementa de manera significativa cuando se pasa del valor del capital humano al VRR, valor más apropiado, puesto que se basa en las preferencias individuales y no en un criterio de cuentas nacionales. Basándonos en el VRR, el costo externo en daños personales que impone un automovilista asciende a $\$ 63.500$ o $\$ 2,6$ por kilómetro. Adicionando el resto de los costos, el costo externo total que genera un vehículo promedio es de $\$ 96.500$, es decir, un costo por kilómetro igual a $\$ 3,9 .{ }^{22}$

Si nos centramos sólo en los costos de daños a las personas, este valor asciende a \$ 127 mil millones. El total de los costos externos asciende a \$193 mil millones. ${ }^{23}$ De este total, $\$ 41$ mil millones se requieren para compensar a los usuarios desprotegidos, $\$ 77$ mil millones para cubrir costos externos de accidentes, una porción ha de incluir costos administrativos y márgenes de ganancia de las compañías de seguro, y el remanente habría de ser apropiado por alguna autoridad de transporte o seguridad vial para ser invertidos en obras de seguridad vial o para compensar a las víctimas de accidentes que pertenecen a la categoría automóvil. ${ }^{24}$ Así, habría una mayor aceptación por parte de la población de estos impuestos pigouvianos.

En cuanto a los subsidios para los usuarios desprotegidos, el monto promedio por persona (suponiendo que las potenciales víctimas son 15 millones de

\footnotetext{
${ }^{22} \mathrm{Si}$ la elasticidad del riesgo de muerte en relación al tráfico en áreas urbanas fuese $-0,2$, la tarifa promedio del SOAP debería costar $\$ 89.100$ por vehículo (\$3,6 por kilómetro), incluyendo todos los costos de los efectos externos y $\$ 53.600$ por vehículo (\$2,2 por kilómetro), considerando sólo los costos de la disposición al pago.

${ }^{23}$ Este valor representa el $34 \%$ del total de los costos de accidentes viales.

${ }^{24} \mathrm{La}$ teoría económica no es clara en cuanto a qué destino a dar a dichos fondos. Una alternativa sugerida es que reemplacen el uso de impuestos que generan una pérdida de "peso muerto" significativa
} 
personas, aproximadamente el total de la población del país) ascendería a $\$ 2.750$, una cifra mínima desde el punto de vista individual. Parece más sensato compensar ex post sólo a aquellos usuarios de categorías débiles que sean victimas de un accidente.

El total de los costos percibidos por los usuarios (viales y desprotegidos) del sistema de transporte asciende a $\$ 488$ mil millones y deben ser asumidos por ellos mismos. En caso que estos usuarios sean aversos al riesgo, recurrirán a la compra de pólizas de seguros que los cubran en casos de accidente. ${ }^{25}$ En Chile, los montos totales vendidos en pólizas de seguro para vehículos motorizados ascienden a \$131,5 mil millones en el año 2000. ${ }^{26}$ Para peatones sólo existe el seguro de accidentes personales, que entrega una compensación en caso de muerte accidental, incapacidad total o permanente y reembolso de gastos médicos a causa de accidentes que puedan ocurrir en el desempeño de la profesión o en la vida privada. Este seguro vendió pólizas por un total de \$3 mil millones en el año 2000, por lo que el valor correspondiente a peatones sería menor. ${ }^{27}$

A fin de una comparación internacional, tomemos como referencia el valor reportado por Lindberg (2001) para Suecia. Lindberg estima la prima de seguros obligatorios promedio por vehículo en una cifra superior a los \$300.000 ( $€ 391)$, incluyendo el total de los costos internos y externos de los accidentes. Esta diferencia se debe, principalmente, al VRR utilizado, diez veces superior al considerado por nosotros (ver Lindberg, 2001, Cuadro 8).

Si quisiéramos establecer un impuesto pigouviano óptimo a fin de internalizar las externalidades de accidentes viales, la tarifa óptima sería menor que los valores estimados, en la medida que la curva de costos medios sea creciente tal como se ilustra en el Gráfico 1. Para determinar la tarifa de equilibrio, el ideal sería conocer la función de demanda. Desde el punto de vista práctico esto es complejo, por lo que se debería proceder por prueba y error.

Para completar esta sección, analicemos qué papel desempeña el Seguro Obligatorio de Accidentes Personales (SOAP) en Chile en la internalización de las externalidades de accidentes viales. El SOAP es de carácter obligatorio (Ley 18.490) y tiene como finalidad indemnizar a las personas que resulten lesionadas en un accidente en que participen vehículos asegurados o a los beneficiarios de aquéllos que perezcan a raíz de un accidente de ese tipo. Con fecha 18 de agosto del año 2003 fue publicada la Ley 19.887, que modifica la Ley 18.490. Esta nueva ley introdujo una serie de modificaciones; una de ellas elevó a UF 300 la compensación monetaria para los beneficiarios de una víctima fatal. Esta y otras modificacio-

\footnotetext{
${ }^{25}$ La suma de los costos totales externos y los costos totales percibidos supera al total de los costos de accidentes. Debe recordarse que por costos externos nos referimos a los costos que un usuario particular impone al resto de los agentes económicos.

${ }^{26}$ Fuente: Asociación de Aseguradores de Chile, Boletín Estadístico, Vehículos Motorizados: 1993-2003. Este valor excluye la recaudación proveniente del Seguro Obligatorio de Accidentes Personales.

${ }^{27}$ Fuente: Asociación de Aseguradores de Chile, Boletín Estadístico, Seguros de Accidentes Personales: 1993-2003.
} 
nes han hecho incrementar las primas del SOAP a partir del año 2004, fecha de entrada en vigencia de la ley.

\section{GRAFICO 1}

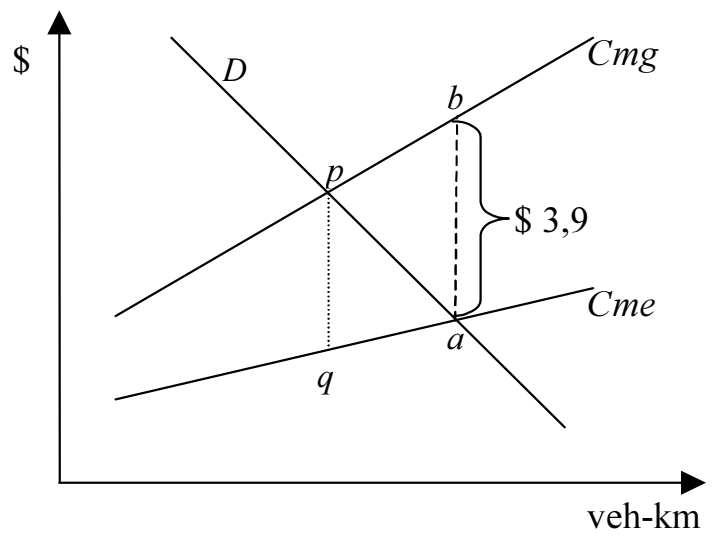

El segmento $\overline{a b}$ representa la diferencia actual entre el costo marginal $(\mathrm{Cmg})$ y el costo medio percibido ( $\mathrm{Cme}$ ) de accidentes. En el equilibrio oferta ( $\mathrm{Cmg}$ )-demanda $(D)$, la tarifa se reducirá al valor dado por el segmento $\overline{p q}$.

Con la modificación a la ley, la prima promedio por vehículo se duplicó a casi $\$ 15.000^{28}$, totalizando una recaudación superior a los $\$ 30$ mil millones a partir del año 2004. Si comparamos estos valores con los cálculos de los actuales costos externos de accidentes según el Cuadro 2, se cubrirían sólo los costos externos en daños personales utilizando el valor del capital humano como base del costo en daños personales. Esto es, sin embargo, pura coincidencia, puesto que el criterio utilizado para calcular las primas del SOAP no se basa en la metodología desarrollada en este documento, sino en prorratear las compensaciones que la ley otorga a las víctimas de los accidentes entre todos los vehículos del país. Lamentablemente, el SOAP es un cargo fijo que se paga una vez al año y no guarda relación con los kilómetros recorridos ni con el historial de accidentes o estilo de conducción del conductor. Tal como se lo cobra, es percibido como un impuesto más por usar un vehículo y no tiene ningún impacto como herramienta de gestión de seguridad vial.

\footnotetext{
${ }^{28}$ Fuente: Asociación de Aseguradores de Chile, boletín estadístico, SOAP 1994-2004.
} 


\section{CONCLusiones}

Hemos presentado un modelo simple de accidentes tanto para corrientes de tráfico homogéneo como para corrientes de tráfico mixto; en ambos casos se generan externalidades de accidentes y, por lo tanto, se debe establecer algún tipo de impuesto pigouviano para su internalización. Los dos elementos clave para calcular la magnitud de los costos de las externalidades de accidentes son la elasticidad del riesgo de accidentes y el valor monetario de reducir una víctima fatal y una víctima grave. Dado este valor, la determinación de los costos es bastante sencilla.

A fin de corregir las fallas de mercado señaladas, se consideró como la mejor opción el cobro de primas de seguro obligatorio por kilómetro. Así, las consecuencias de las conductas de los automovilistas sobre los riesgos de accidentes de terceros serían internalizadas. Estas primas habrían de influir en las decisiones de elección de vehículo, de estilo de conducción y de cuántos kilómetros circular. Entre otros, este tipo de tarifas debería propiciar el uso de vehículos más amigables con peatones, ciclistas u otras categorías de vehículos motorizados débiles; este cargo constituiría una señal para toda la industria en el diseño de vehículos más seguros para terceros agentes. El lector debe tener presente que la finalidad de este impuesto pigouviano no es otorgar una compensación a las víctimas de los accidentes viales, sino procurar el nivel óptimo de seguridad vial. A efectos de reparación o compensación a las víctimas, éstas deben recurrir a la compra de pólizas de seguro.

Se determinaron los costos externos actuales de accidentes viales en Chile. Incluyendo los costos de la disposición al pago, daños materiales y costos de terceros agentes, dicho valor ascendería a $\$ 96.500$ por vehículo o $\$ 3,9$ por kilómetro circulado. Con su diseño actual, el SOAP no constituye una efectiva herramienta de gestión económica de seguridad vial.

Se analizó también si bajo condiciones de mercado las empresas automotrices y proveedoras de infraestructura vial disponen de adecuados incentivos para proveer el nivel óptimo de seguridad en sus productos. Debido a ciertas imperfecciones de mercado, estas empresas no dispondrían de los incentivos adecuados para invertir en la seguridad de sus productos. El cobro de primas de seguros por kilómetro induciría, de manera indirecta, a las empresas automotrices a brindar los niveles óptimos de seguridad. En relación a los proveedores de infraestructura vial, aquella tarifa no lograría su objetivo y debería contemplarse el cobro de algún cargo a los proveedores.

Para cerrar el presente documento, se señalan algunas posibles extensiones de este trabajo a fin de mejorar el análisis. Desde el punto de vista empírico, deberían: a) estimarse las diferentes elasticidades riesgo de accidentes en relación al tráfico para el medio chileno (es de esperar que estos valores puedan diferir de los obtenidos en Europa y EE.UU.); b) lograr una mejor comprensión de la relación congestión-seguridad vial, y c) afinar la estimación de los valores monetarios por reducir una muerte y un herido grave. Los cálculos deberían también discriminar 
por tipo de vehículo, tipos de vía e idealmente por tipo de conductor. En cuanto al cálculo de los costos externos de accidentes, deberían incluirse los costos en mayores tiempos de viaje que genera conducir con mayor precaución.

\section{REFERENCIAS}

Baumol, W. y J. Oates (1988), The Theory of Environmental Policy, Segunda Edición, Cambridge University Press.

Bertín-Jones, M. (2003), "Improving Vehicle Safety Using a Market-Based Methodology", Traffic Engineering and Control, 44(6): 206-208.

CITRA (1996), Investigación Diseño de Programa de Seguridad Vial Nacional, para el Ministerio de Transportes y Telecomunicaciones y Ministerio de Obras Públicas de Chile.

Elvik, R. (1994), “The External Costs of Traffic Injury: Definition, Estimation, and Possibilities for Internalization", Accident Analysis and Prevention, 26: 719-732.

Fridstrøm, L. (1999), Econometric Models of Road Use, Accident, and Road Investment Decisions. Tesis de Doctorado, Institute of Transport Economics, TØI report 457/1999, University of Oslo, Oslo.

Fridstrøm, L., J. Ifver, S. Ingebrigtsen, R. Kulmala y L. Thomsen (1995), "Measuring the Contribution of Randomness, Exposure, Weather and Daylight to the Variation in Road Accident Counts", Accident Analysis and Prevention, 27: $1-20$.

Hojman, P., J. de D. Ortúzar y L.I. Rizzi (2005), “On the Joint Valuation of Averting Fatal Victims and Severe Injuries in Highway Accidents", Journal of Safety Research, (por aparecer).

Jansson, J.O. (1994), "Accident Externality Charges", Journal of Transport Economics and Policy, 28: 31-43.

Jones Lee, M., D. O’Reilly y P. Philips (1993), “The Value of Preventing Non-Fatal Road Injuries: Findings of a Willingness-to-pay National Sample Survey", TRL, Working Paper SRC2, Transport Research Laboratory, Crowthorne.

Jones Lee, M. y G. Loomes (2003), "Valuation of Safety", en D. Hensher,. and K. Button (Eds.) Handbook No. 4 Transport and the Environment. Macmillan, New York.

Lindberg, G. (2001), "Traffic Insurance and Accident Externality Charges", Journal of Transport Economics and Policy, 35: 399-416.

Lindberg, G.; P. Ajo, A.B. da Silva, C. Crawford, R. Krupp, M.R. Osório Nunes, J. Peirson, G. Schneglberger y W. Smolders (1999), Calculating Transport Accident Costs, mimeo, European Commission.

Litman, T.A. (2004), "Pay-as-you-Drive Pricing for Insurance Affordability", mimeo, Victoria Transport Policy Institute.

OECD (1990), Behavioural Adaptations to Changes in the Road Transport System, París. 
Peltzman S. C. (1975), “The Effects of Automobile Safety Regulation”, Journal of Political Economy, 83: 677-725.

Persson, U. y K. Ödegaard (1995), "External Cost Estimates of Road Traffic Accidents", Journal of Transport Economics and Policy, 29: 291-304.

Peterson, S., G. Hoffer y E. Millner (1995), “Are Drivers of Air-bag Equipped Car More Aggresive? A Test of the Offsetting Behavior Hypothesis", Journal of Law and Economics, 37: 251-264.

Rizzi, L.I. y J. de D. Ortúzar (2003), "Stated Preference in the Evaluation of Interurban Road Safety", Accident Analysis and Prevention, 35: 9 - 22.

Rizzi, L.I. y J. de D. Ortúzar (2005), "Road Safety Valuation under a Stated Choice Framework", Journal of Transport Economics and Policy, (por aparecer).

Shavell, E. (1984), "A Model of the Optimal Use of Liability and Safety Regulation", Rand Journal of Economics, 15: 271-280.

Shavell, E. (2003), "Economic Analysis of Law", Working Paper 9694, National Bureau of Economic Research, Cambridge.

SNRA (1989), Swedish Nacional Road Administration's EVA Manual. Borlänge.

Tay, R. (2002), "The Prisoner's Dilemma and Vehicle Safety: Some Policy Implications", Journal of Transport Economics and Policy, 36: 491-495.

Wilde, G. (2000), Enhancing Workers' and Drivers' Interest in their Own Safety, mimeo, Department of Psychology, Queens University, Ontario, Canadá. 